
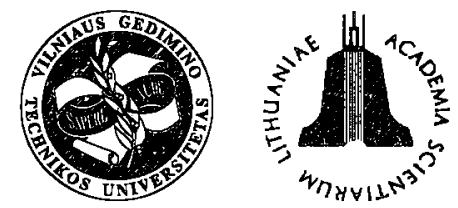

ISSN 1648-4142 TRANSPORT

http:/www.vtu.lt/english/editions

TRANSPORT - 2003, Vol XVIII, No 4, 153-156

\title{
THE METHODOLOGY OF MODELLING TAXI RANK SERVICE
}

\author{
Jonas Butkevičius ${ }^{1}$, Algimantas Juozapavičius ${ }^{2}$ \\ ${ }^{1}$ Dept of Transport Management, Vilnius Gediminas Technical University, \\ Plytines g. 27, LT-2016Vilnius, Lithuania.E-mail:vladas@ti.vtu.lt \\ ${ }^{2}$ Dept of Computer Science II, Vilnius University, Naugarduko g. 24, LT-2006 Vilnius, Lithuania \\ E-mail: algimantas.juozapavicius@maf.vu.lt
}

Received 200301 31; accepted 20030630

\begin{abstract}
The operation of a taxi rank as a passengers' transportation service was not researched till now by means of scientific methods. Authors of this article are suggesting the methodology for the analysis and evaluation of a taxi rank as a passengers' transportation system, when passengers pick up a taxi at taxi ranks. According to the analysis of the collected data the assumption has been made that the number of passengers coming to a taxi rank, as well as the number of taxis arriving to take passengers at a rank are distributed by Poisson distribution law controlled by specific values of parameters. This observation enables us to consider a taxi rank as a queueing system, having behavior controlled by two parameters: the expected number of taxis arriving to the rank at a time period and the expected number of passengers picking up a taxi at a rank at the same period of time. Usually values of these parameters do not coincide what makes the modelling of a taxi rank a valuable queueing system.

Theoretical considerations as well as practical formulas of this article lead us to the methodology of modelling the demand for taxis at a taxi rank.

The methodology suggested enables to estimate the efficiency of taxi rank functioning from different points of view. A taxi company may evaluate the costs related to the outage time of a taxi, as well as the amount of taxis in a rank satisfying the demand of passengers for transportation.
\end{abstract}

Keywords: urban public transport, transportation by taxi, passengers' service, taxi rank, Poisson distribution, queueing system.

\section{Introduction}

The organization of transportation of passengers by car, and especially by taxi is an important issue of city transportation system.

The passengers' transportation by taxi was analyzed by many scientists in different countries - Cassidy R. C., Kobza J. E. [1], Desaulniers G., Villeneuve D. [2], Friesz T., Bernstein D., Stough R. [3], Bhat C. R. [4], Jacoby H. G. [5], Mc Laren J. [6], Sans B., Milot L. [7].

The system of passengers' transportation by taxi in Lithuania, after regaining the independence, is facing extraordinary development. This system of transportation however is based on "primitive market" principles, which can be characterized by the following aspects:

taxi companies hire drivers "from the street" together with their autos, making no investment into car renovation, and when such a driver wears and tears his car, the company hires another driver;
- by reason of this, taxi companies increase transportation prices, fighting for market;

- some taxi companies grab taxi ranks at their disposition in the most favorable regions of the city (in the downtown of the city, at department stores, at places of people concentration, etc.) using illegal methods;

- city municipalities have no power to struggle licenses of taxi companies;

- the organization of passengers' transportation by taxi is based on no scientific or research methods, etc.

The potential passenger has two possibilities to pick up a taxi: to have a telephone call or to pick up taxi at a taxi rank. These two options have to be modelled and analyzed in order to make right principles of organization of transportation by taxi.

In this article the authors are analyzing the organization of passengers' transportation by taxi, when passenger picks up taxi at a taxi rank. This important task is not analyzed enough in our country yet [8]. 


\section{The Stochastic Model of a Demand for Passengers' Transportation by Taxi}

While organizing the entire system of passengers' transportation by taxi it is essential to know consistent patterns of taxis coming to a taxi rank. The analysis of experimental data and observations lead to the proposition that the period of time for taxi arrivals to a taxi rank may be defined on the basis of exponential distribution with the density:

$$
f(t)= \begin{cases}A e^{-M t}, t \geq 0 \\ 0, & t<0\end{cases}
$$

where $M=\frac{1}{2}$ is a parameter, corresponding to the density of distribution. This parameter defines a statistical average number of taxis coming to a taxi rank within the entity of time; $t$ - an average entity of time.

Because of such assumption the taxi rank may be considered as a queueing system. In this case the number $x$ of arriving cars to the rank in time period $t$ is distributed by Poisson distribution law. The taxi arrival probability $x=m$ within period of time $t$ may be expressed by the formula:

$$
P\left(x=\begin{array}{c}
m \\
t
\end{array}\right)={ }_{m !}^{(M t)^{m}} e^{-M t}, m=0,1, \ldots .
$$

On the other hand, the distribution of passengers picking up taxis at a taxi rank may be defined by Poisson distribution law also. The probability of passengers' arrival to a taxi rank in the period of time $t$ may be expressed by the formula:

$$
P\left(y=\begin{array}{c}
m \\
t
\end{array}\right)=\frac{(\lambda \cdot t)^{m}}{m !} e^{\lambda t}, m=0,1, \ldots,
$$

here $\lambda$ - demand of passengers for a taxi, or in other words, the density of passengers coming to a taxi rank in a unit of time.

The number of taxis coming to a taxi rank and the number of passengers demanding a taxi usually do not coincide, so the functionality of a taxi rank really corresponds to a queueing system, which may be used for the evaluation of efficiency of taxi operation. The parameters of such a system, introduced above are indicating the situation, when the demand for a taxi is high (what leads to lines of passengers waiting for a taxi) or the supply of cars is high (what leads to lines of taxis in a rank).

The probability of a number $m$ of taxis arriving to the rank in a time period is described by the formula:

$$
P(x=m)=\int_{0}^{\infty} \frac{(\lambda \cdot t)^{m}}{m !} \cdot e^{-\lambda t} \cdot M e^{-M t} \cdot d t=
$$

$$
\begin{aligned}
& \frac{M \cdot \lambda^{m}}{m !} \int_{0}^{\infty} t^{m} \cdot e^{(\lambda+M) t} \cdot d t= \\
& \frac{M}{\lambda+M}\left(\begin{array}{c}
\lambda \\
\lambda+M
\end{array}\right)^{m}, m=0,1, \ldots
\end{aligned}
$$

This formula corresponds to geometric distribution with parameters $M$ and $(\lambda+M)$. The probability for amount of taxis $m=0,1, \ldots$ in a taxi rank for geometric distribution is given by the expression:

$$
P m=(1-P)^{m} P,
$$

where

$$
P=\frac{M}{\lambda+M} .
$$

These formulas naturally lead to a suggestion that the value of the fulfillment of the demand of passengers' transportation by a taxi may be considered the conditional period of time of demand satisfaction.

The taxi company, optimizing its expenditures and profit is interested to evaluate the optimal taxi line in a taxi rank, $i$ e. the optimal number of taxis waiting for passengers.

As it was established above the distribution of the number of taxis, arriving to a rank and picked up by the specific demand of passengers is expressed by a geometric distribution law. It follows that this probability may be calculated by the formula:

$$
P_{j}=(1-P)^{j+1}\left(1-\frac{P}{1-(1-P)^{u+1}}\right),
$$

where $j=1,2, \ldots, u$, and $u$ - limitary number of taxis in a line (the maximal number of taxis in a rank), in units.

The average number of taxis in a taxi rank is:

$$
\bar{Z}=\sum_{i=1}^{u} P_{j} \cdot j
$$

The loss of a taxi company for the outage of a taxi in a taxi rank during 1 working hour is:

$$
\Delta S_{\text {nuost.val }}=Z \cdot I_{\text {prid }}, \mathrm{Lt} / \mathrm{h},
$$

where $I_{\text {prid }}$ - overhead cost of taxi working hour, Lt/h.

The loss of driving a taxi without a passenger is:

$$
\Delta S_{\text {be kel }}=l_{k e l . j s} \cdot I_{k m}, \mathrm{Lt} \text {, }
$$

where $l_{\text {kel.is }}$ - the distance of driving a taxi before a passenger is picked up, $\mathrm{km} ; I_{k m}-$ cost price of a run, Lt. The cost price of a run for $1 \mathrm{~km}$ is: 


$$
I_{k m}=I_{k \text { int }}+\frac{I_{\text {prid }}}{V_{T}}, \mathrm{Lt},
$$

where $V_{T}$ - technical velocity of a taxi, $\mathrm{km} / \mathrm{h}$.

The average number of a taxi leaving a rank without passengers in a period of time when passengers do not pick up a taxi is:

$$
\dddot{Y}=\sum_{i=1}^{\infty} P_{u+i}^{i} \text {. }
$$

One of the most important indicators, representing the quality of transportation by taxi service, is the gratification of demand for transportation. It is important therefore to define a period of time, when a passenger will find a taxi waiting for a passenger at a rank.

This period of time may be expressed by the parameter of a waiting mean. For given distribution the waiting mean of a period of time, when a taxi will wait for a passenger while there are $n$ cars in a taxi rank, is calculated by the formula:

$$
M[n]=\frac{1-P}{P} n=\frac{\lambda}{M} n .
$$

The average value of such a waiting mean expressed in minutes is:

$$
M\left[T_{n}\right]=\ddot{t}_{\text {int }} M[n] .
$$

The observation and the data collected by the authors for a waiting mean at a taxi rank allow us to define a suitable number of taxis at a rank, satisfying the demand of passengers for transportation.

This determination benefits for twofold directions - passengers have less time to wait for a taxi, and taxi companies can reduce their costs because of shorter outages of a taxi in a taxi rank.

\section{The Methodology of Modelling Transportation Demand for Passengers at a Taxi Rank}

The theoretical considerations and explicit formulas suggested and investigated above allow us to formulate the methodology of modelling the demand for a system of passengers' transportation by a taxi at a taxi rank.

In order to determine or forecast lines of taxis waiting for passengers or passengers waiting for a taxi at a taxi rank it is necessary to produce empirical observations for the determination of taxi flow intensity $M$ and passengers' transportation demand $\lambda$ values for various periods of daytime. Then the comparison of values of such parameters enables us to forecast the formation or decline taxi or passengers lines at a taxi rank (Fig).

The methodology enables us to evaluate the efficiency of operation of a taxi rank by methods of a queueing system. The evaluation may be made from the point of view of a taxi company, or from some other aspects. The formalized assertions and formulas $(9,12)$ enable us to calculate the outage time of a taxi and the costs related to these outages.

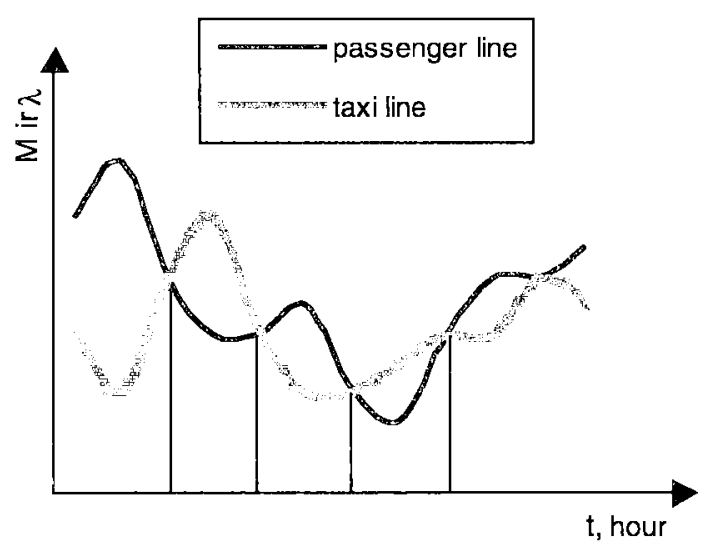

The formation and decline of taxi and passenger lines according to empirical observations

This methodology enables us to evaluate the input of time, when the increasing flow of passengers diminishes lines of taxis waiting for passengers.

\section{Conclusions}

1. While organizing the system of passengers' transportation by car - taxi, it is important to know regular laws of taxis and passengers arriving to a taxi rank. The authors determined that the amount of taxis arriving to a taxi rank as well as the amount of passengers in a rank obey Poisson distribution law with specific parameters.

2. Usually the number of cars at a taxi rank and the number of passengers waiting for a taxi do not coincide. Because of this the formalism of a queueing system may be applied to model the operation of a taxi rank.

3. The theoretical assumptions and explicit formulas suggested and analyzed in this article enable us to formalize and adopt the methodology, modelling the demand for a taxi at a taxi rank and to deduce suitable parameters governing the operation of a taxi rank as a system.

4. In order to evaluate or forecast lines of taxis or passengers at a taxi rank at different periods of day time it is necessary to evaluate or empirically deduce the values of the intensity of taxi flow $M$ and the values of the intensity of passengers' flow $\lambda$ for various periods of day time. The comparison of such values enables us to forecast the formation or diminishing of lines of taxis or passengers at a taxi rank.

5 . The methodology enables us to evaluate the efficiency of taxi rank operation as a queueing system from 
many points of view, for example, from the point of view of a taxi company. The formalism suggested gives a possibility to calculate the outage time of taxis and related losses.

6. The research done by the authors enables to determine the average mean of waiting time of taxis at a taxi rank corresponding to the existing demand for a taxi and fulfill it.

\section{References}

1. Cassidy, R. C.; Kobza, J. E. A Probabilistic Approach to Evaluate Strategis for Selecting a Parking Sapce. Transportation Science, Vol 32, 1998, p. 30-42.

2. Desaulniers, G.; Villeneuve, D. The Shortest Path Problem with Time Windows and Linear Waiting Costs. Transportation Science, Vol 34, 2000, p. 313-320.

3. Friesz, T.; Bernstein, D.; Stough, R. Dynamic Systems, Variational Inequalities and Control Theoretic Models for
Proedicting Time-varuing Urban Network Flows. Transportation Science, Vol 30, 1996, p. 14-31.

4. Bhat, C. R. Incorporating Observed and Unobserved Heterogeneity in Urban Work Travel Mode Choice Modelling Transportation Science, Vol 34, 2000, p. 228-239.

5. Jacoby, H. G. Access to Markets and the Benefits of Rural Roads. The Economic Journal, Vol 110, No 465, Blackwell Publichers Ltd Oxford, UK and Boston, USA, 2000, p. 713 737.

6. Mc Laren, J. Black Markets and Optimal Evadable Taxation. The Economic Journal, Vol 108, No 448, Blackwell Publichers Ltd Oxford, UK and Boston, USA, 1998, p. 665679.

7. Sans, B.; Milot, L. Performability of a Congested Urban Transportation Network when Accident Information is Available. Transportation Science, Vol 33, 1999, p. 67-79.

8. Butkevičius, J. Modelling of the depedence of travel frequency on the lenght duration of a jorney. Improvement of city passenger transport systems. Transport, Vol XVII, No 6. Vilnius: Technika, 2002, p. 223-225. 